\title{
Levels of Urinary Metabolites of Organophosphate Flame Retardants, TDCIPP, and TPHP, in Pregnant Women in Shanghai
}

\author{
Liping Feng, ${ }_{1}^{1}$ Fengxiu Ouyang, ${ }^{2}$ Liangpo Liu, ${ }^{3}$ Xu Wang, ${ }^{2}$ Xia Wang, ${ }^{2}$ Yi-Ju Li, \\ Amy Murtha, ${ }^{1}$ Heqing Shen, ${ }^{3}$ Junfeng Zhang, ${ }^{5,6}$ and Jun Jim Zhang ${ }^{2}$ \\ ${ }^{1}$ Department of Obstetrics and Gynecology and Duke Global Health Institute, Duke University, Durham, NC, USA \\ ${ }^{2}$ MOE-Shanghai Key Laboratory of Children's Environmental Health, Xinhua Hospital, School of Medicine, \\ Shanghai Jiao Tong University, Shanghai, China \\ ${ }^{3}$ Key Lab of Urban Environment and Health, Institute of Urban Environment, Chinese Academy of Sciences, Xiamen, China \\ ${ }^{4}$ Department of Biostatistics and Bioinformatics, Duke University, Durham, NC, USA \\ ${ }^{5}$ Nicholas School of the Environment and Duke Global Health Institute, Duke University, Durham, NC, USA \\ ${ }^{6}$ Duke Kunshan University, Kunshan, China
}

Correspondence should be addressed to Liping Feng; feng0007@mc.duke.edu

Received 22 July 2016; Revised 26 October 2016; Accepted 1 December 2016

Academic Editor: Chunrong Jia

Copyright (C) 2016 Liping Feng et al. This is an open access article distributed under the Creative Commons Attribution License, which permits unrestricted use, distribution, and reproduction in any medium, provided the original work is properly cited.

Flame retardants are widely used in consumer products to reduce their flammability. Previously used flame retardants have been sequentially banned due to their environmental and human toxicity. Currently, tris(1,3-dichloropropyl) phosphate (TDCIPP) and triphenyl phosphate (TPHP) are among the most commonly used flame retardants. TDCIPP and TPHP are reproductive toxins and have carcinogenic, neurotoxic, and endocrine-disrupting properties. Although high levels of TDCIPP and TPHP have been found in drinking water, seawater, and office air in China, data regarding human exposure are lacking. In this study, we assessed the level of urinary TPHP and TDCIPP metabolites (DPHP and BDCIPP, resp.) in a cohort of pregnant women $(N=23)$ from Shanghai, China, using liquid chromatography-tandem mass spectrometry. DPHP were detected in $100 \%$ urine samples, while only four urine samples had detectable level of BDCIPP in this cohort (17\% detected). Geometric means of DPHP and BDCIPP concentrations were $1.1 \mathrm{ng} / \mathrm{mL}$ (interquartile range [IQR]: $0.6,1.5 \mathrm{ng} / \mathrm{mL}$ ) and $1.2 \mathrm{ng} / \mathrm{mL}$ (IQR: $0.6,2.2 \mathrm{ng} / \mathrm{mL}$ ), respectively. In this small cohort, urinary DPHP and BDCIPP levels were not significantly correlated with miscarriages, neonatal birthweight, gestational diabetes, or maternal age. These data suggest that exposure to TPHP is widespread, and they demonstrate the feasibility of using urinary biomarkers to measure exposures to modern flame-retardant chemicals.

\section{Introduction}

Flame retardants are widely used in consumer products such as textiles, plastics, furniture, electronics, cars, and construction materials to meet flammability standards since the 1960 s [1]. Previously used flame retardants have been sequentially banned due to their environmental and human toxicity, including endocrine disruption, neurotoxicity, and carcinogenicity. Until recently, polybrominated diphenyl ethers (PBDEs) accounted for a large proportion of flame retardants used in household products $[1,2]$. However, in the past several years, common PBDE mixtures have been banned or voluntarily phased-out in the United States and elsewhere [3]. Thus, the use of alternative flame retardants has been on the rise [2]. Organophosphate flame retardants (OPFRs) such as tris (1,3-dichloropropyl) phosphate (TDCIPP) and triphenyl phosphate (TPHP) are now among the most commonly used flame retardants $[1,4-6]$. These flame retardants trends are similar in China. Based on China Flame Retardant Industry Report, 2012-2015, China has been a large producer and supplier of flame retardant in the world, with the output in 2011-2012 approximating 800,000-900,000 tons. Chlorinated 
and brominated flame retardants are still taking a lion's share in Chinese flame retardant market, with the proportion as high as $50 \%$ or so. Meanwhile, China fire retardant industry is upgrading product mix and technologies so as to follow the global development trend of halogen-free flame retardants. Strikingly, OPFRs have seen rapid development and grown into a hot variety, with major producers including Jiangsu Yoke Technology, Zhejiang Wansheng Co., Ltd, which are close to Shanghai. A recent study demonstrated the declined emission of PBDEs from industries in Southern China [7]. Consequently, ubiquitous environmental exposures of TDCIPP and TPHP have been reported in the United States [6, 8-17], Europe [18-22], and China [23-26].

TDCIPP and TPHP are persistent and bioaccumulative in the environment [27]. Toxicological data suggest that TDCIPP and TPHP are reproductive toxins $[28,29]$ and have carcinogenic [30], neurotoxic [31-33] and endocrinedisrupting properties [34-39]. Currently, available data are very limited on human exposure $[10,11,40-42]$ and data on potential human health effects are lacking. To the best of our knowledge, data regarding human exposure to TPHP and TDCIPP in China have not been reported. Epidemiologic studies are critically needed. In our present pilot work, we utilized recently developed methods to extract and measure bis(1,3-dichloropropyl) phosphate (BDCIPP) and diphenyl phosphate (DPHP), the respective metabolites of TDCIPP and TPHP, in urine samples from a group of pregnant women in Shanghai.

\section{Materials and Methods}

2.1. Study Population. We recruited 23 pregnant women during November 2015 at the Xinhua Hospital, affiliated with Shanghai Jiao Tong University School of Medicine. Women provided demographic information and a spot urine sample during their second trimester clinical visit. Our final sample consisted of 23 urine specimens. All study protocols were approved by the institutional review board at the Xinhua Hospital, and all women provided informed consent.

2.2. Urinary TDCIPP and TPHP Metabolites Analysis. Urine samples were collected in sterile polyethylene specimen containers. Samples were packed on ice and transported to the lab in Xinhua Hospital, where they were aliquoted into $15 \mathrm{~mL}$ conical centrifuge tubes and frozen at $-80^{\circ} \mathrm{C}$ until shipping. After all samples were collected, urine samples were packed on dry ice and transported to Institute of Urban Environment (IUE), Chinese Academy of Sciences in Xiamen, China for sample analysis. Modified extraction and measurement methods described previously $[40,41]$ were used. DPHP and d10-DPHP were purchased from TRC (Toronto, Canada). BDCIPP and d10-BDCIPP were purchased from Wellington Laboratories (Guelph, Ontario, Canada). Briefly, after thawing, $5 \mathrm{~mL}$ urine was buffered to $\mathrm{pH}=6.5$ with $1 \mathrm{M}$ acetic acid if the samples were above $\mathrm{pH}$ 6.5. Urine samples were then spiked with $50 \mu \mathrm{L}$ mixture of working internal standard, d10-BDCPP and d10-DPP, solution $(500 \mathrm{ng} / \mathrm{mL})$. The internal standards were deconjugated using $5 \mu \mathrm{L}$ of $\beta$-glucuronidase/sulfatase from Helix pomatia (Type HP-2, aqueous solution, $\beta$ glucuronidase activity $\geq 100000$ units $/ \mathrm{mL}$, sulfatase activity $\leq 7500$ units $/ \mathrm{mL}$, Sigma-Aldrich, USA). The samples were incubated at $37^{\circ} \mathrm{C}$ for $90 \mathrm{~min}$ to deconjugate. A mixedmode anion exchange solid phase extraction (Strata-X-AW, $60 \mathrm{mg} / 3 \mathrm{~mL}$, Phenomenex Inc., Torrance, CA, USA) was preconditioned with dichloromethane $(3 \mathrm{~mL})$ and methanol $(3 \mathrm{~mL})$ sequentially. The treated urine was loaded onto the cartridge at a rate of $1 \mathrm{~mL} / \mathrm{min}$. After the loading of treated mixture, the SPE column was cleaned up with $3 \mathrm{~mL}$ distilled water. The analytes were eluted with methanol $(3 \mathrm{~mL})$ at $1 \mathrm{~mL} / \mathrm{min}$. The eluate was evaporated to dryness under a gentle stream of nitrogen at $45^{\circ} \mathrm{C}$ and then resuspended in $500 \mu \mathrm{L}$ methanol for the further analysis.

The target compounds were separated by an Accela UHPLC pumping system (Thermo Fisher Scientific, SanJosé, USA), coupled with an Accela Autosampler and Degasser. Separation of the compounds was carried out on a Hypersil Gold aQ C18 column $(1.9 \mu \mathrm{m}, 100 \mathrm{~mm} \times 2.1 \mathrm{~mm}$, Thermo Fisher Scientific) which was kept at $30^{\circ} \mathrm{C}$. The mobile phase of water and methanol was pumped at a flow rate of $0.3 \mathrm{~mL} / \mathrm{min}$. Optimized separation of BDCIPP and DPHP was obtained by using a linear gradient. The gradient was as follows: $0 \mathrm{~min}$, $20 \% \mathrm{~B}$; 0-6 min, $100 \%$ B (linear); hold for $2 \mathrm{~min}$; 8-8.1 min, 20\% B (linear); and hold for $9.9 \mathrm{~min}$. The total run time for each injection was $(18 \mathrm{~min})$ and the injection volume was $20 \mu \mathrm{L}$.

The target compounds were analyzed by a triple quadrupole mass analyzer (TSQ Vantage, Thermo Fisher Scientific) which was fitted with atmospheric pressure chemical ionization in negative ion mode. The following working conditions were applied: spray voltage at $2.5(-) \mathrm{kV}$; vaporizer and capillary temperature at 300 and $325^{\circ} \mathrm{C}$, respectively; sheath and auxiliary gas at 45 and 15 arbitrary units (a.u.), respectively; cycle time of 1.0 second. Argon pressure in the collision cell (Q2) was set at 1.5 mTorr and the mass resolution at the first (Q1) and third (Q3) quadrupole was set at $0.70 \mathrm{Da}$ at full width at $50 \%$ of maximum (FWHM). Precursor ion, S-lens RF amplitude, and collision energy (CE) in Q2 were optimized individually per compound and/or transition (Table 1). Quantification and confirmation data were acquired in selected reaction monitoring (SRM) mode, and the transitions are displayed as Table 1. Instrument control and data processing were carried out by means of Xcalibur Software 2.2 SP 1.48 (Thermo Electron, San José, USA).

For quality assurance purposes we evaluated the recovery of d10-BDCIPP and d10-DPHP in spiked pooled samples and measured the amount of BDCIPP and DPHP levels in laboratory blanks $(n=3)$. The laboratory blank was deionized water obtained from a Millipore water purification system (ELGA LabWater, Lane End, HP14 3JH, UK). The deionized water was further cleaned up by SPE as real sample extraction before being used to prepare the buffer solutions and other aqueous solutions. The average recoveries of d10-BDCIPP and d10-DPHP were $112.5 \pm 0.6$ and $81.1 \pm 6.6 \%$, respectively. Very small amounts of DPHP were detected in laboratory blanks $(0.012 \mathrm{ng} / \mathrm{mL})$, while BDCIPP was detected in relatively higher levels in laboratory blanks $(0.13 \mathrm{ng} / \mathrm{mL})$. The 
TABLE 1: Parameters for MRM acquisition of the analysis.

\begin{tabular}{|c|c|c|c|c|c|c|c|}
\hline Analyte & Detection mode & Precursor ion $(m / z)$ & Slens $(\mathrm{Hz})$ & $\mathrm{SRM}^{*}(m / z)$ & Collision energy $(\mathrm{eV})$ & SRM2 $(m / z)$ & Collision energy $(\mathrm{eV})$ \\
\hline BDCIPP & - & 319 & 59 & 35 & 55 & 37 & 14 \\
\hline $\mathrm{D}_{10}$-BDCIPP & - & 329 & 57 & 35 & 63 & l & l \\
\hline DPHP & - & 249 & 79 & 93 & 37 & 155 & 25 \\
\hline $\mathrm{D}_{10}$-DPHP & - & 259 & 79 & 98 & 40 & l & l \\
\hline
\end{tabular}

${ }^{*}$ Quantitative ions.

TABLE 2: Distribution of urinary DPHP and BDCIPP concentrations $(\mathrm{ng} / \mathrm{mL})$ among 23 urine samples from 23 pregnant women in Shanghai.

\begin{tabular}{|c|c|c|c|c|c|c|c|c|}
\hline \multirow{2}{*}{ Urinary metabolite } & \multirow{2}{*}{ Percent detect ${ }^{*}$} & \multirow{2}{*}{ Geometric mean ${ }^{\#}$} & \multicolumn{5}{|c|}{ Percentiles } & \multirow{2}{*}{ Maximum } \\
\hline & & & 10th & 25 th & 50th & 75 th & 95th & \\
\hline DPHP & 100 & 1.1 & 0.45 & 0.59 & 0.83 & 1.48 & 5.92 & 7.3 \\
\hline BDCIPP & 17 & 1.2 & 0.43 & 0.59 & 1.58 & 2.17 & 2.20 & 2.2 \\
\hline
\end{tabular}

${ }^{*}$ Percentage based on 23 urine samples analyzed for DPHP and BDCIPP.

\# Geometric mean was calculated based on detectable concentrations.

method detection limit (MDL) or limit of detection (LOD) was calculated using three times the standard deviation of the blanks, which was $0.057 \mathrm{ng} / \mathrm{mL}$ for DPHP and $0.11 \mathrm{ng} / \mathrm{mL}$ for BDCIPP. Levels of BDCIPP and DPHP in urine were corrected for recovery of the mass labeled internal standards. All concentration data were corrected with laboratory blanks. If subtracting the blank value resulted in a negative value, the concentration of the sample was regarded as below MDL. We have chosen to present results using the MDL/ $\sqrt{2}$ method for values below the MDL [40]. Sample specific gravity (SG) was measured in each urine sample prior to analysis using a digital handheld refractometer (Atago Co., Ltd., Tokyo, Japan). All data analyses were conducted using specific gravity adjusted concentrations account for urine dilution [40].

2.3. Statistical Analyses. We calculated descriptive statistics for each metabolite, including the geometric mean concentration and selected percentiles (10th, 25th, 50th, 75th, and 90th percentiles). We also examined the distribution of urinary BDCIPP and DPHP graphically. Due to the small sample size and the nonnormality of BDCIPP and DPHP, the exact Wilcoxon rank-sum test was used to compare the median differences of urinary BDCIPP or DPHP concentrations between 3 miscarriages and 15 term pregnancies. The same test was also used to compare the median differences of urinary BDCIPP or DPHP concentrations between subjects with/without gestational diabetes. Multivariable logistic regression modeling was performed to test the effect of DPHP on gestational diabetes with adjustment of neonatal birthweight. The pairwise Spearman correlation for urinary BDCIPP or DPHP with maternal age and neonatal birthweight were computed and tested. The correlation of BDCIPP and DPHP levels among subjects was also tested.

The level of significance was a two-sided $p$ value $<0.05$. All analyses were performed using the SAS 9.4 software (SAS Institute, Inc., Cary, NC, USA).

\section{Results}

Twenty-three women participating in our study ranged in age from 25 to 40 years at conception and all had a college education and above. Prepregnancy body mass index of all subjects is in the range of 18-25. Subjects' occupation and life style information was not detailed enough to indicate sources of exposure. Three subjects had elective abortions. Five subjects could not be followed up for birth outcomes. One subject delivered a macrosomia infant. All other subjects ( $n=14)$ delivered healthy, full-term infants. However, among the 15 subjects, 4 had gestational diabetes and 1 had preeclampsia during pregnancy.

DPHP was detected in all urine samples (100\% detected); four urine samples had detectable level of BDCIPP (17\% detected). A lower bound of 0.16 was used for those subjects with nondetectable urinary BDCIPP based on the MDL. The low detect rate of urinary BDCIPP in this cohort could be caused by the higher detection limit of BDCIPP and matrix effect in urine sample in our study. Urinary concentrations of these compounds varied from individual to individual in detectable samples. The distribution of urinary BDCIPP and DPHP concentrations is shown in Table 2 and Figure 1. Levels of BDCIPP and DPHP were not correlated in this cohort $(r=-0.2, p=0.4)$. The geomean of DPHP or BDCIPP is higher in patients who had miscarriages than subjects who delivered at full term (mean [SD]: 1.3 [1.4] versus 2.0 [2.2] for DPHP; 0.4 [0.6] versus 0.8 [1.1] for BDCIPP). However, the median of these compounds is similar between these two groups ( 0.8 versus 0.7 for DPHP and 0.2 versus 0.2 for BDCIPP). And no comparisons are statistically significant $(p>0.5)$. The mean and median of DPHP are higher in patients with gestational diabetes than those without gestational diabetes (mean (SD): 2.6 (2.4) versus 1.4 (1.7) and median: 1.8 versus 0.8 ). However, median differences of urinary BDCIPP or DPHP concentrations between pregnancy complicated with/without gestational diabetes were not significantly different $(p=0.6$ for BDCIPP and $p=0.2$ for DPHP). Since birthweight for DPHP is close to nominal significance for gestational diabetes, we also performed multivariable logistic regression modeling to test the effect of DPHP on gestational diabetes with adjustment of birthweight; it remained nonsignificant (odds ratio [OR] 4.6, $95 \%$ confidence interval $[\mathrm{CI}]: 0.16-133.8, p=0.4$ ). No significant correlation was observed between urinary BDCIPP 


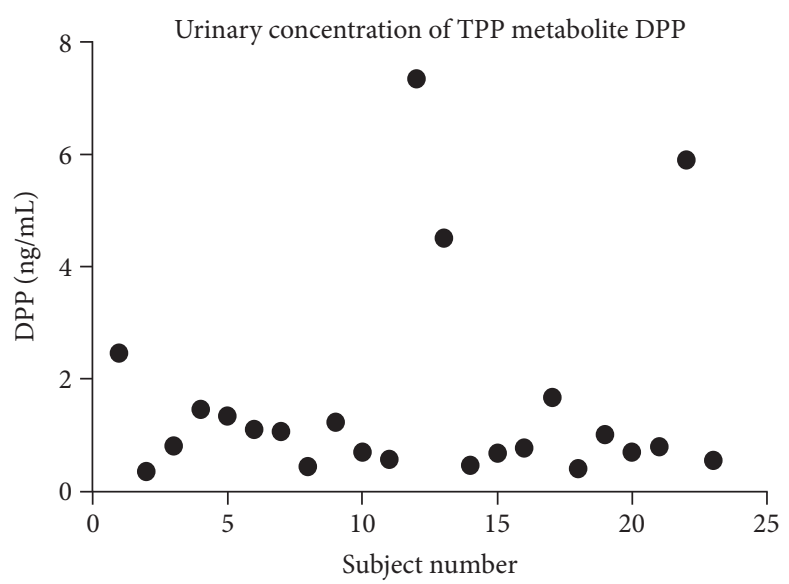

(a)

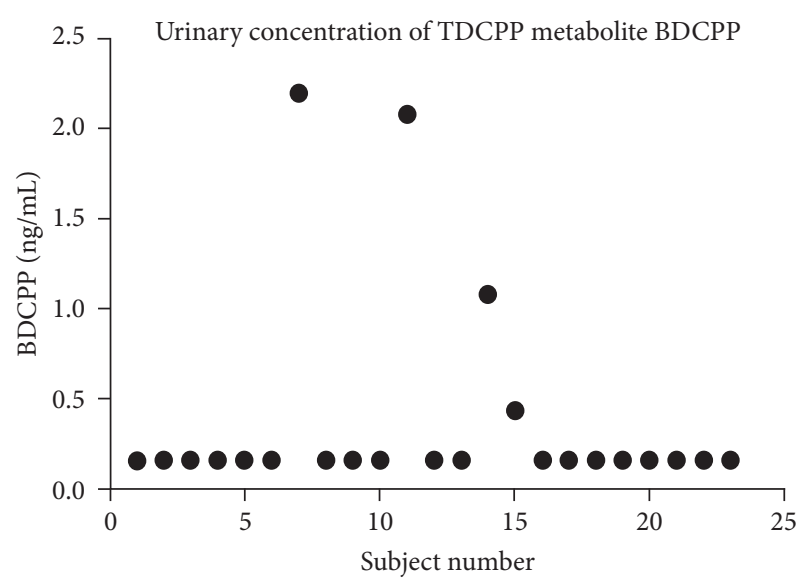

(b)

FIGURE 1: Distribution of urinary concentration of TPHP and TDCIPP metabolites DPHP and BDCIPP, respectively.

or DPHP with maternal age $(r=0.2, p=0.4$ for BDCIPP; $r=-0.16, p=0.5$ for DPHP) and neonatal birthweight $(r=-0.3, p=0.2$ for BDCIPP; $r=0.2, p=0.45$ for DPHP). Detailed statistical analysis data are presented in the Supplementary Data (in Supplementary Material available online at http://dx.doi.org/10.1155/2016/9416054).

\section{Discussion}

DPHP was detectable in all urine samples collected, suggesting possible widespread exposure to DPHP itself or its parent compounds including TPHP in this study region, while exposures to TDCIPP might be selective, with only a $17 \%$ detectable rate in pregnant women in Shanghai. These results for TPHP are consistent with several small studies from the United States that also reported relatively variable but near ubiquitous exposure in nonpregnant populations $[10,11,41]$. However, the geometric mean concentrations were higher in this Shanghai pregnant cohort than in nonpregnant subjects. The results in this Shanghai pregnant cohort are similar to those recently reported in pregnant women in the United States (US) [40] and Canada [42]. In the Canadian cohort of pregnant women, the detection frequency of BDCIPP (29.2\%) is also significantly less than DPHP (91.7\%), which is similar to our observation. The geomean concentration of DPHP urinary levels $(2.72 \mathrm{ng} / \mathrm{mL})$ observed in Canadian pregnant cohort is higher than the US $(1.9 \mathrm{ng} / \mathrm{mL})$ and our Shanghai pregnant cohort $(1.1 \mathrm{ng} / \mathrm{mL})$. In contrast, the geomean concentration of BDCIPP urinary levels $(0.27 \mathrm{ng} / \mathrm{mL})$ observed in Canadian pregnant cohort is lower than the US $(1.1 \mathrm{ng} / \mathrm{mL})$ and our Shanghai pregnant cohort $(1.2 \mathrm{ng} / \mathrm{mL})[40,42]$. The authors of the US study suggest that differences in excretion rates and kidney function during pregnancy may explain the higher metabolite levels observed in pregnant women relative to nonpregnant cohorts [40]. However, the relatively similar geomean of BDCIPP levels between the Canadian pregnant cohort and the other nonpregnant cohorts indicates that the measurements among pregnant women may be more reflective of actual exposures rather than confounding factors of altered excretion associated with pregnancy stage [42].

We observed nonstatistically significant correlations between urinary DPHP and BDCIPP concentrations, which may be explained by the higher detection limit of BDCIPP and matrix effect in urine sample in our study. This observation may also be explained by differences in the sources of exposure to TDCIPP and TPHP. All subjects in our study cohort were neither living near fire retardant industries nor factory workers, so we suspect the source of exposure in this cohort are unlikely to be direct industrial plant exposures. Data relating to OPFRs in China remain scarce [25]. Few studies that reported fairly ubiquitous environmental exposures of TDCIPP and TPHP in drinking water [23], seawater [25], and office air [24] do not explain the selective exposure of TDCIPP in this study cohort. While both TDCIPP and TPHP are used as additive flame retardants in household products, TPHP is also used as a plasticizer and lubricant and in hydraulic fluids [1], which may contribute to the more ubiquitous human exposures in this cohort. Differences in the metabolism and excretion of TDCIPP and TPHP also provide possible explanations for the nonsignificant correlation observed in urinary biomarkers of exposure [40]. Interestingly, two recent studies demonstrated a route of exposure of TDCIPP and TPHP through diet and food consumptions in China $[43,44]$. The differences in the levels of TDCIPP and TPHP in foods and dietary differences among subjects might in part contribute to the observed discrepancy in urinary DPHP and BDCIPP concentrations in our study.

Although the toxicokinetics of TDCIPP and TPHP in the human body have yet to be explored, data suggest that they are rapidly metabolized to (BDCIPP and DPHP) and are rapidly eliminated from the body $[41,45,46]$. Nonetheless, a previous study observed moderate to strong reliability in the levels of BDCIPP and DPHP in urine samples collected throughout pregnancy and shortly after giving birth [40]. Therefore, the study authors concluded that a single measure of levels during pregnancy may be sufficient in characterizing levels throughout pregnancy [40]. These data suggest 
that TDCIPP and TPHP may come from more continuous sources of exposure than diet, such as contaminated dust in the home or workplace environments. This would seem a reasonable hypothesis given that human exposure to $\mathrm{PBDE}$ flame retardants in the United States has been demonstrated to occur primarily from exposure to household dust, both in adults and in children [5, 47].

Adjustments for urine dilution have been recommended in the assessment of xenobiotics and are commonly included in epidemiologic investigations [48]. In this study, we chose to adjust with urinary specific gravity, which is thought to be less impacted by changes with age, body composition, physical activity, urine flow, time of day, diet, disease, and pregnancy than other measures such as creatinine $[48,49]$. However, as a previous study suggested, other measures of urine dilution and methods of correction should be included in future studies of BDCIPP and DPHP. This study is also limited by the small sample size ( 23 women with a total of 23 measurements) and composition-specifically highlyeducated and normal-weight pregnant women-which may differ substantially from other populations. Sample size may be one of the explanations of the observed nonstatically significant association of levels of urinary TDCIPP and TDD with subject characteristics and pregnancy outcomes. We observed a trend of negative association of TDD exposure with subject age. However, it did not reach statistical significance. The other limitation of this study is that the subjects' occupation and life style information was not detailed enough to indicate sources of exposure. Follow-up of our findings in larger cohorts with longitudinal data will provide additional insights.

\section{Conclusions}

Our results demonstrate ubiquitous exposure to TPHP (100\% detection of TPHP metabolite DPHP) and exposure to TDCIPP (17\% above-detection limit of the urinary metabolite BDCIPP) in pregnant women is likely in Shanghai, China. Additionally, we observed a modest degree of variability in urinary metabolites of BDCIPP and DPHP among women in our study population. The association of urinary levels of BDCIPP and DPHP with pregnancy outcomes and subject characteristics was not observed, as our analyses were limited by our small sample size and relatively homogeneous study population of Shanghai pregnant women. This is the first feasibility study showing that exposure to modern flameretardant chemicals can be detected in pregnant women living in Shanghai. Further studies can use these urinary biomarkers of exposure to help identify sources of exposure to OPFRs and/or assess OPFRs' impact on health outcomes in a larger cohort.

\section{Abbreviations}

BDCIPP: Bis(1,3-dichloropropyl) phosphate

CI: $\quad$ Confidence interval

DPHP: Diphenyl phosphate

IQR: Interquartile range

MDL: Method detection limit
OPFRs: Organophosphate flame retardants

PBDEs: Polybrominated diphenyl ethers

SG: $\quad$ Specific gravity

TDCIPP: Tris(1,3-dichloropropyl) phosphate

TPHP: Triphenyl phosphate.

\section{Disclosure}

Liping Feng and Fengxiu Ouyang are co-first authors.

\section{Competing Interests}

The authors declare they have no competing financial interests with respect to this manuscript, or its content, or subject matter.

\section{Acknowledgments}

This study was funded by the Chairman's Research Fund of the Department of Obstetrics and Gynecology, Duke University. This study was also supported by Ministry of Science and Technology of China (2014CB943300) and the National Natural Science Foundation of China (no. 81372954).

\section{References}

[1] I. van der Veen and J. de Boer, "Phosphorus flame retardants: properties, production, environmental occurrence, toxicity and analysis," Chemosphere, vol. 88, no. 10, pp. 1119-1153, 2012.

[2] H. M. Stapleton, J. G. Allen, S. M. Kelly et al., "Alternate and new brominated flame retardants detected in U.S. house dust," Environmental Science and Technology, vol. 42, no. 18, pp. 69106916, 2008.

[3] H. Fromme, G. Becher, B. Hilger, and W. Völkel, "Brominated flame retardants-exposure and risk assessment for the general population," International Journal of Hygiene and Environmental Health, vol. 219, no. 1, pp. 1-23, 2016.

[4] H. M. Stapleton, S. Klosterhaus, S. Eagle et al., "Detection of organophosphate flame retardants in furniture foam and U.S. house dust," Environmental Science and Technology, vol. 43, no. 19, pp. 7490-7495, 2009.

[5] H. M. Stapleton, S. Eagle, A. Sjödin, and T. F. Webster, "Serum PBDEs in a North Carolina toddler cohort: associations with handwipes, house dust, and socioeconomic variables," Environmental Health Perspectives, vol. 120, no. 7, pp. 1049-1054, 2012.

[6] H. M. Stapleton, S. Sharma, G. Getzinger et al., "Novel and high volume use flame retardants in US couches reflective of the 2005 PentaBDE phase out," Environmental Science and Technology, vol. 46, no. 24, pp. 13432-13439, 2012.

[7] Q. Wu, H. Li, D. T. Kuo et al., "Occurrence of PBDEs and alternative halogenated flame retardants in sewage sludge from the industrial city of Guangzhou, China," Environmental Pollution, vol. 220, pp. 63-71, 2017.

[8] H. M. Stapleton, S. Klosterhaus, A. Keller et al., "Identification of flame retardants in polyurethane foam collected from baby products," Environmental Science and Technology, vol. 45, no. 12, pp. 5323-5331, 2011.

[9] J. D. Meeker and H. M. Stapleton, "House dust concentrations of organophosphate flame retardants in relation to hormone 
levels and semen quality parameters," Environmental Health Perspectives, vol. 118, no. 3, pp. 318-323, 2010.

[10] C. C. Carignan, M. D. McClean, E. M. Cooper et al., "Predictors of tris(1,3-dichloro-2-propyl) phosphate metabolite in the urine of office workers," Environment International, vol. 55, pp. 56-61, 2013.

[11] J. D. Meeker, E. M. Cooper, H. M. Stapleton, and R. Hauser, "Urinary metabolites of organophosphate flame retardants: temporal variability and correlations with house dust concentrations," Environmental Health Perspectives, vol. 121, no. 5, pp. 580-585, 2013.

[12] E. D. Schreder, N. Uding, and M. J. La Guardia, "Inhalation a significant exposure route for chlorinated organophosphate flame retardants," Chemosphere, vol. 150, pp. 499-504, 2016.

[13] K. A. Maruya, N. G. Dodder, A. Sengupta et al., "Multimedia screening of contaminants of emerging concern (CECS) in coastal urban watersheds in southern California (USA)," Environmental Toxicology and Chemistry, vol. 35, no. 8, pp. 19861994, 2016.

[14] M. J. La Guardia and R. C. Hale, "Halogenated flame-retardant concentrations in settled dust, respirable and inhalable particulates and polyurethane foam at gymnastic training facilities and residences," Environment International, vol. 79, pp. 106-114, 2015.

[15] A. S. Keller, N. P. Raju, T. F. Webster, and H. M. Stapleton, "Flame retardant applications in camping tents and potential exposure," Environmental Science \& Technology Letters, vol. 1, no. 2, pp. 152-155, 2014.

[16] A. Sengupta, J. M. Lyons, D. J. Smith et al., "The occurrence and fate of chemicals of emerging concern in coastal urban rivers receiving discharge of treated municipal wastewater effluent," Environmental Toxicology and Chemistry, vol.33, no. 2, pp. 350358, 2014.

[17] J. G. Allen, H. M. Stapleton, J. Vallarino et al., "Exposure to flame retardant chemicals on commercial airplanes," Environmental Health, vol. 12, article no. 17, 2013.

[18] A. M. Sundkvist, U. Olofsson, and P. Haglund, "Organophosphorus flame retardants and plasticizers in marine and fresh water biota and in human milk," Journal of Environmental Monitoring, vol. 12, no. 4, pp. 943-951, 2010.

[19] J. A. Andresen, A. Grundmann, and K. Bester, "Organophosphorus flame retardants and plasticisers in surface waters," Science of the Total Environment, vol. 332, no. 1-3, pp. 155-166, 2004.

[20] A. Marklund, B. Andersson, and P. Haglund, "Organophosphorus flame retardants and plasticizers in Swedish sewage treatment plants," Environmental Science and Technology, vol. 39, no. 19, pp. 7423-7429, 2005.

[21] H. Wolschke, R. Sühring, Z. Xie, and R. Ebinghaus, "Organophosphorus flame retardants and plasticizers in the aquatic environment: a case study of the Elbe River, Germany," Environmental Pollution, vol. 206, pp. 488-493, 2015.

[22] S. Brommer, S. Harrad, N. Van Den Eede, and A. Covaci, "Concentrations of organophosphate esters and brominated flame retardants in German indoor dust samples," Journal of Environmental Monitoring, vol. 14, no. 9, pp. 2482-2487, 2012.

[23] J. Li, N. Yu, B. Zhang et al., "Occurrence of organophosphate flame retardants in drinking water from China," Water Research, vol. 54, pp. 53-61, 2014.

[24] Z. Cao, F. Xu, A. Covaci et al., "Differences in the seasonal variation of brominated and phosphorus flame retardants in office dust," Environment International, vol. 65, pp. 100-106, 2014.

[25] M. Hu, J. Li, B. Zhang, Q. Cui, S. Wei, and H. Yu, "Regional distribution of halogenated organophosphate flame retardants in seawater samples from three coastal cities in China," Marine Pollution Bulletin, vol. 86, no. 1-2, pp. 569-574, 2014.

[26] F. Yang, J. Ding, W. Huang, W. Xie, and W. Liu, "Particle sizespecific distributions and preliminary exposure assessments of organophosphate flame retardants in office air particulate matter," Environmental Science and Technology, vol. 48, no. 1, pp. 63-70, 2014.

[27] L. V. Dishaw, D. L. Hunter, B. Padnos, S. Padilla, and H. M. Stapleton, "Developmental exposure to organophosphate flame retardants elicits overt toxicity and alters behavior in early life stage zebrafish (Danio rerio)," Toxicological Sciences, vol. 142, no. 2, pp. 445-454, 2014.

[28] S. P. McGee, E. M. Cooper, H. M. Stapleton, and D. C. Volz, "Early zebrafish embryogenesis is susceptible to developmental TDCPP exposure," Environmental Health Perspectives, vol. 120, no. 11, pp. 1585-1591, 2012.

[29] J. Fu, J. Han, B. Zhou et al., "Toxicogenomic responses of zebrafish embryos/larvae to tris(1,3-dichloro-2- propyl) phosphate (TDCPP) reveal possible molecular mechanisms of developmental toxicity," Environmental Science \& Technology, vol. 47, no. 18, pp. 10574-10582, 2013.

[30] R. I. Freudenthal and R. T. Henrich, "Chronic toxicity and carcinogenic potential of tris-(1,3,-dichloro-2-propyl) phosphate in Sprague-Dawley rat," International Journal of Toxicology, vol. 19, no. 2, pp. 119-125, 2000.

[31] Q. Wang, J. C.-W. Lam, Y.-C. Man et al., "Bioconcentration, metabolism and neurotoxicity of the organophorous flame retardant 1,3-dichloro 2-propyl phosphate (TDCPP) to zebrafish," Aquatic Toxicology, vol. 158, pp. 108-115, 2015.

[32] L. Yuan, J. Li, J. Zha, and Z. Wang, "Targeting neurotrophic factors and their receptors, but not cholinesterase or neurotransmitter, in the neurotoxicity of TDCPP in Chinese rare minnow adults (Gobiocypris rarus)," Environmental Pollution, vol. 208, pp. 670-677, 2016.

[33] L. V. Dishaw, C. M. Powers, I. T. Ryde et al., "Is the PentaBDE replacement, tris (1,3-dichloro-2-propyl) phosphate (TDCPP), a developmental neurotoxicant? Studies in PC12 cells," Toxicology and Applied Pharmacology, vol. 256, no. 3, pp. 281-289, 2011.

[34] Q. Wang, J. C. W. Lam, J. Han et al., "Developmental exposure to the organophosphorus flame retardant tris(1,3-dichloro-2propyl) phosphate: estrogenic activity, endocrine disruption and reproductive effects on zebrafish," Aquatic Toxicology, vol. 160, pp. 163-171, 2015.

[35] Q. Zhang, M. Lu, X. Dong et al., "Potential estrogenic effects of phosphorus-containing flame retardants," Environmental Science and Technology, vol. 48, no. 12, pp. 6995-7001, 2014.

[36] A. Farhat, J. K. Buick, A. Williams et al., “Tris(1,3-dichloro-2propyl) phosphate perturbs the expression of genes involved in immune response and lipid and steroid metabolism in chicken embryos," Toxicology and Applied Pharmacology, vol. 275, no. 2, pp. 104-112, 2014.

[37] X. Liu, K. Ji, A. Jo, H.-B. Moon, and K. Choi, "Effects of TDCPP or TPP on gene transcriptions and hormones of HPG axis, and their consequences on reproduction in adult zebrafish (Danio rerio)," Aquatic Toxicology, vol. 134-135, pp. 104-111, 2013.

[38] S. Kim, J. Jung, I. Lee, D. Jung, H. Youn, and K. Choi, “Thyroid disruption by triphenyl phosphate, an organophosphate flame 
retardant, in zebrafish (Danio rerio) embryos/larvae, and in GH3 and FRTL-5 cell lines," Aquatic Toxicology, vol. 160, pp. 188-196, 2015.

[39] Q. Wang, K. Liang, J. Liu et al., "Exposure of zebrafish embryos/ larvae to TDCPP alters concentrations of thyroid hormones and transcriptions of genes involved in the hypothalamic-pituitarythyroid axis," Aquatic Toxicology, vol. 126, pp. 207-213, 2013.

[40] K. Hoffman, J. L. Daniels, and H. M. Stapleton, "Urinary metabolites of organophosphate flame retardants and their variability in pregnant women," Environment International, vol. 63, pp. 169-172, 2014.

[41] E. M. Cooper, A. Covaci, A. L. N. van Nuijs, T. F. Webster, and H. M. Stapleton, "Analysis of the flame retardant metabolites bis(1,3-dichloro-2-propyl) phosphate (BDCPP) and diphenyl phosphate (DPP) in urine using liquid chromatographytandem mass spectrometry," Analytical and Bioanalytical Chemistry, vol. 401, no. 7, pp. 2123-2132, 2011.

[42] I. Kosarac, C. Kubwabo, and W. G. Foster, "Quantitative determination of nine urinary metabolites of organophosphate flame retardants using solid phase extraction and ultra performance liquid chromatography coupled to tandem mass spectrometry (UPLC-MS/MS)," Journal of Chromatography B: Analytical Technologies in the Biomedical and Life Sciences, vol. 1014, pp. 24-30, 2016.

[43] X. Zhang, W. Zou, L. Mu et al., "Rice ingestion is a major pathway for human exposure to organophosphate flame retardants (OPFRs) in China," Journal of Hazardous Materials, vol. 318, pp. 686-693, 2016.

[44] Y. Ma, K. Cui, F. Zeng et al., "Microwave-assisted extraction combined with gel permeation chromatography and silica gel cleanup followed by gas chromatography-mass spectrometry for the determination of organophosphorus flame retardants and plasticizers in biological samples," Analytica Chimica Acta, vol. 786, pp. 47-53, 2013.

[45] R. K. Lynn, K. Wong, C. Garvie-Gould, and J. M. Kennish, "Disposition of the flame retardant, tris(1,3-dichloro-2-propyl) phosphate, in the rat," Drug Metabolism and Disposition, vol. 9, no. 5, pp. 434-441, 1981.

[46] A. A. Nomeir, S. Kato, and H. B. Matthews, "The metabolism and disposition of tris(1,3-dichloro-2-propyl) phosphate (fyrol FR-2) in the rat," Toxicology and Applied Pharmacology, vol. 57, no. 3, pp. 401-413, 1981.

[47] D. J. Watkins, M. D. McClean, A. J. Fraser et al., "Impact of dust from multiple microenvironments and diet on PentaBDE body burden," Environmental Science and Technology, vol. 46, no. 2, pp. 1192-1200, 2012.

[48] M. F. Boeniger, L. K. Lowry, and J. Rosenberg, "Interpretation of urine results used to assess chemical exposure with emphasis on creatinine adjustments: a review," American Industrial Hygiene Association Journal, vol. 54, no. 10, pp. 615-627, 1993.

[49] J. M. Braun, A. E. Kalkbrenner, A. M. Calafat et al., "Variability and predictors of urinary bisphenol a concentrations during pregnancy," Environmental Health Perspectives, vol. 119, no. 1, pp. 131-137, 2011. 


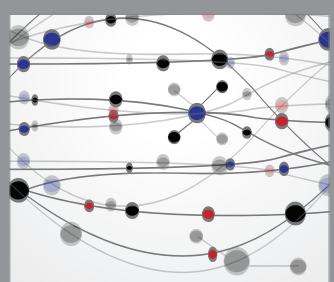

The Scientific World Journal
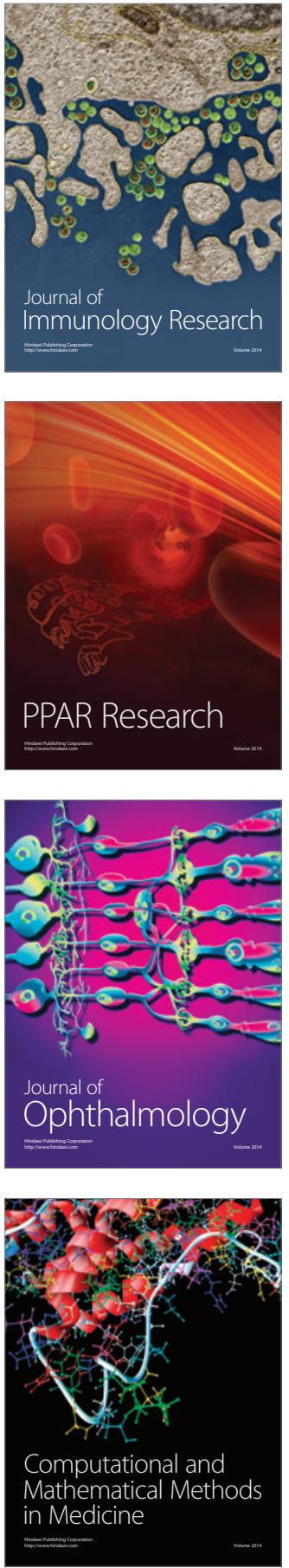

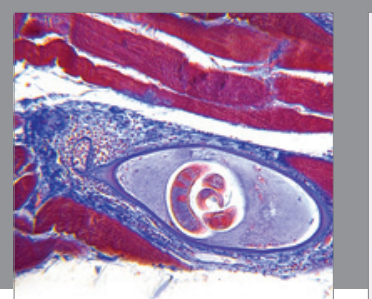

Gastroenterology Research and Practice

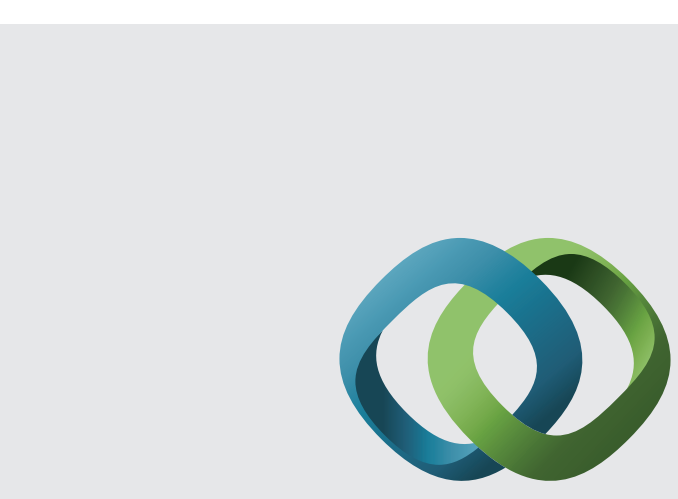

\section{Hindawi}

Submit your manuscripts at

http://www.hindawi.com
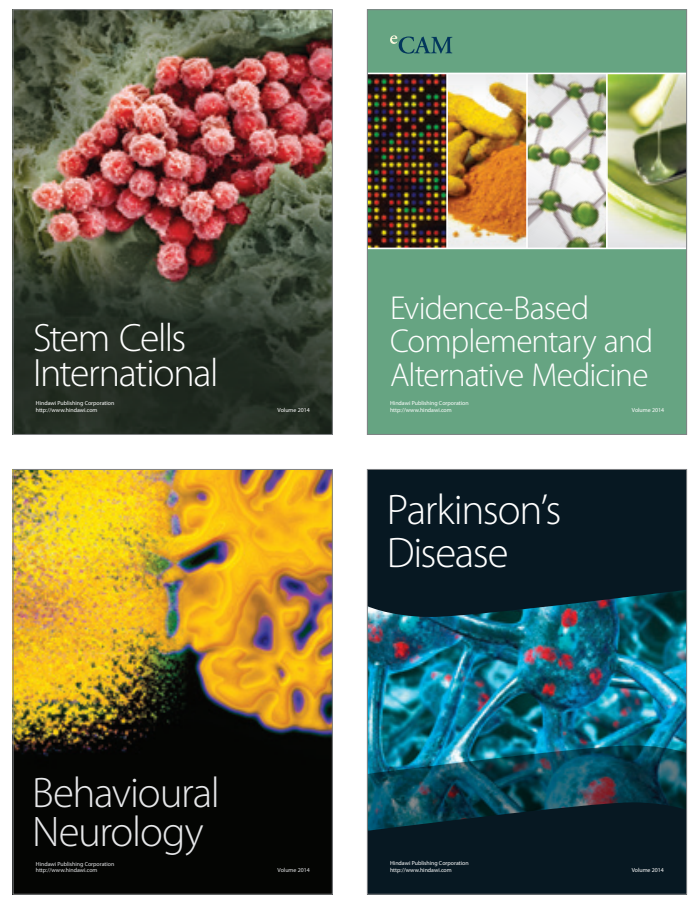
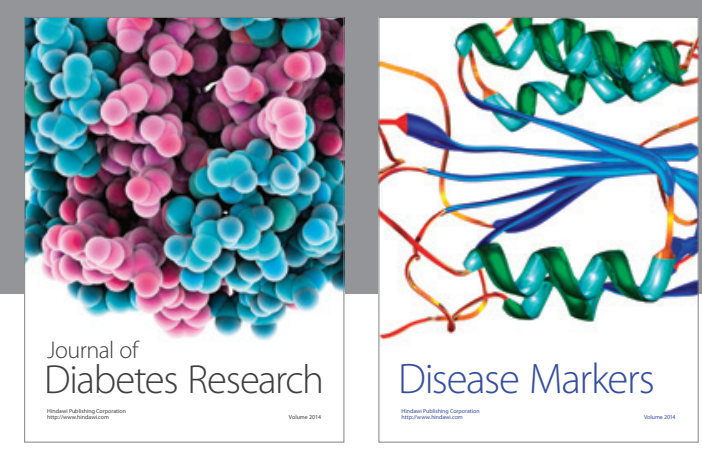

Disease Markers
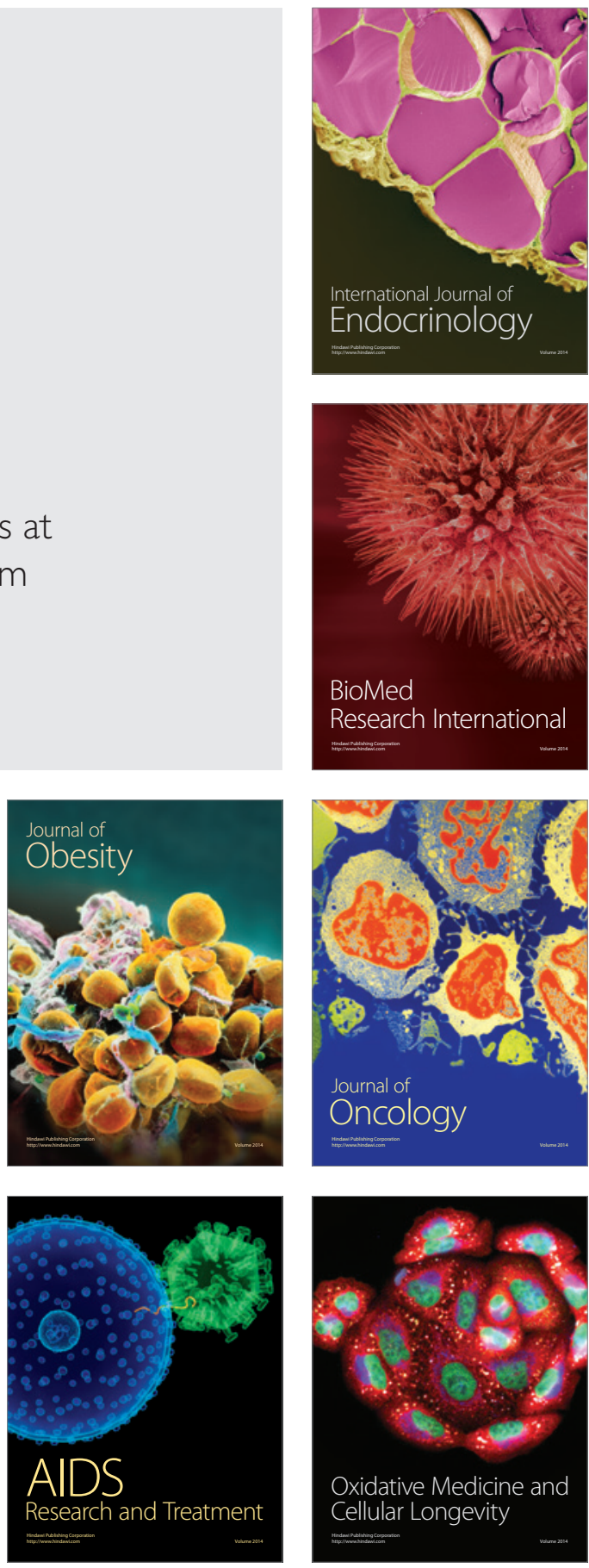